\title{
Primary cardiac angiosarcoma: a rare tumor
}

\begin{abstract}
Angiosarcomas are malignant mesenchymal proliferations of endothelial elements characterized by rapidly proliferating, extensively infiltrating anaplastic cells. Wellknown sites of occurrence include the skin and post-radiation breast tissue. It is exceedingly rare to find angiosarcoma as a cardiac primary. We present a case of primary cardiac angiosarcoma in a 55-year-old African-American female presenting with sudden and dramatic large hemorrhagic pericardial effusion. She had no known prior symptoms. Cardiac MRI revealed a ten centimeter mass involving the right atrium, right ventricle and encasing the right coronary artery, as well as multiple lung nodules. Via pericardial window, biopsies were simultaneously obtained of the cardiac mass and of the lung nodules. Tissue from the cardiac mass showed necrotic cells with noncontributory immunostains. However, tissue from the lung nodule showed viable tumor cells and confirmed primary cardiac angiosarcoma with pulmonary metastases. Immunohistochemical stains on the pulmonary nodule tissue were positive for $\mathrm{CD} 31$, CD34 and vimentin and negative for AE1/AE3, cam5.2, TTF-1, desmin, OCT3/4 and CA125. This case highlights a sudden and dramatic presentation of rare primary cardiac angiosarcoma.
\end{abstract}

Keywords: angiosarcoma, cardiac, primary
Volume 4 Issue 2 - 2017

\author{
A Chen,' J Aziz, ${ }^{2}$ A Toubaji,' S Aziz \\ 'Department of Pathology, George Washington University, USA \\ ${ }^{2}$ Dept. Pharmacology and Physiology, Georgetown University, \\ USA \\ ${ }^{3}$ Division of Cardiovascular Surgery, George Washington \\ University, USA
}

Correspondence: Anne Chen, George Washington University, Department of Pathology, 2I20 L Street, NW, Suite 200-2nd Floor, Gelman Building, Washington, D.C. 20037, Tel (56I)30I5613, Fax (202)677-660I,Email alchen@email.gwu.edu

Received: January 24, 2017 | Published: March 07, 2017
Abbreviations: PCA, primary cardiac angiosarcoma; H\&E, hematoxylin and eosin

\section{Introduction}

Malignant cardiac tumors are rare, with an autopsy prevalence of $0.001-0.28 \% .^{1}$ Benign tumors are more common than malignant, and most initial differential diagnoses for a cardiac mass, when first detected, involve benign lesions. An angiosarcoma is a malignant neoplasm characterized by rapidly proliferating, extensively infiltrating anaplastic cells derived from blood vessels that line irregular blood-filled spaces. ${ }^{2}$ When occurring as a cardiac primary tumor, most cases are detected late due to wide variation in presentation from asymptomatic to aggressive local recurrence and widespread metastases. A high index of suspicion must be maintained to make a diagnosis. Pathological findings and staging usually determine prognosis and therapeutic options. We describe a case of cardiac angiosarcoma, outlining the clinical presentation, radiological and pathology findings.

\section{Case presentation}

A 55-year-old African American woman presented with a onemonth history of progressive exertional dyspnea and chest tightness. She had no significant past medical history. An echocardiogram revealed a large pericardial effusion and features of cardiac tamponade. A cardiac CT scan (Figure 1A) showed a mass at the pericardium extending into the right ventricular myocardium. Multiple bilateral lung nodules were noted with the largest measuring $1.6 \mathrm{~cm}$ in diameter.

A pericardial catheter was placed and a large bloody effusion was drained. A cardiac MRI (Figure 1B) revealed a $10 \mathrm{~cm}$ infiltrating soft tissue mass involving the right atrium and right ventricle. The right coronary artery was encased and attenuated. The mass extended into the atrioventricular groove and infiltrated the roof of the right ventricle. The anterior surface of the aortic root was also encased.
Heterogeneous enhancement of the mass suggested a vascular lesion suggestive of angiosarcoma.

The patient was taken to the operating room where, via a limited right infra-mammary incision and under general anesthesia, pericardial window (Figure 1C) biopsies of the adherent pericardium, the mass invading the right ventricle, and a pulmonary wedge resection of nodules in the right lower lobe were made. Intraoperatively and grossly, the tumor was noted to be markedly firm and dark red in color. Frozen section of tissue from a lung nodule in the right lower lobe showed a high-grade malignant neoplasm with large pleomorphic cells and frequent mitoses.

All biopsy tissues were fixed in $10 \%$ formalin and paraffinembedded. Permanent hematoxylin and eosin (H\&E) sections of the main cardiac mass showed fragments of necrotic tumor with ghost cells. The nuclei were large with nucleoli; however, a definitive diagnosis could not be made from the H\&E sections alone. Immunohistochemical stains were attempted with CD34, CD31 and vimentin but were noncontributory. The pericardial biopsy showed fragments of fibrous tissue with acute and chronic inflammation, granulation tissue and reactive mesothelium. There was a single focus of hyperchromatic cells with crush artifact and high grade cytology, highly suggestive but not diagnostic for a focus of tumor.

However, the wedge resection of the right lower lobe revealed angiosarcoma with lymphovascular invasion. This biopsy showed highly atypical and large malignant cells with increased mitoses (Figure 2A \& Figure 2B). The immunohistochemical stains showed diffuse positivity for CD31, CD34, and vimentin (Figures 3A-3C). Stains for AE1/AE3 (Figure 3D), cam5.2, TTF-1, desmin, and OCT3/4 were negative. Along with the clinical history, a final diagnosis of angiosarcoma of the heart with metastases to the lungs was made.

Due to the extensive cardiac involvement and presence of metastases, surgical resection was not an option, and the patient 
underwent palliative chemotherapy. Unfortunately, the lung lesions rapidly progressed and the patient expired from exsanguinating hemoptysis.

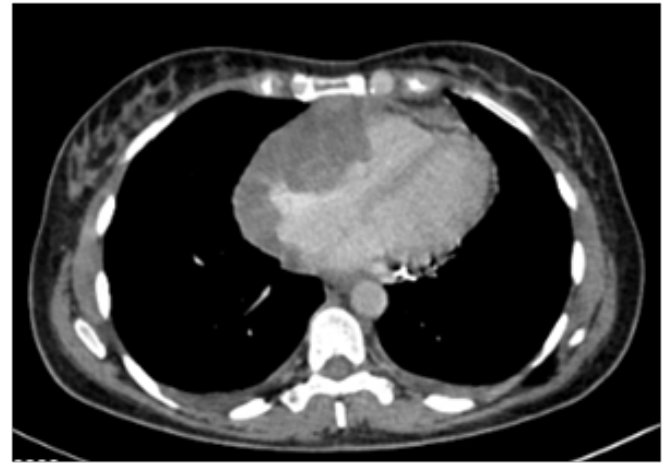

Figure IA CT scan shows a mass at the pericardium extending into the right ventricular myocardium.

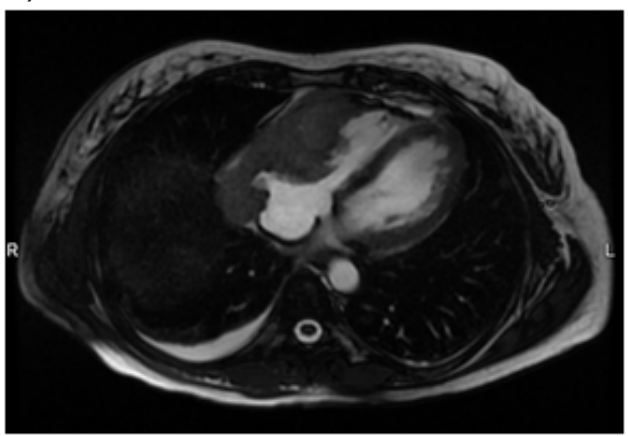

Figure IB Cardiac MRI shows an infiltrating soft tissue mass involving the right atrium and right ventricle.

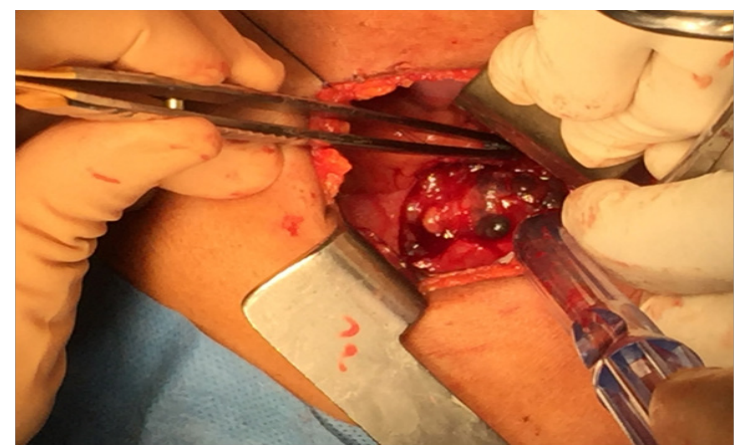

Figure IC: Intraoperative photo showing pericardial window.

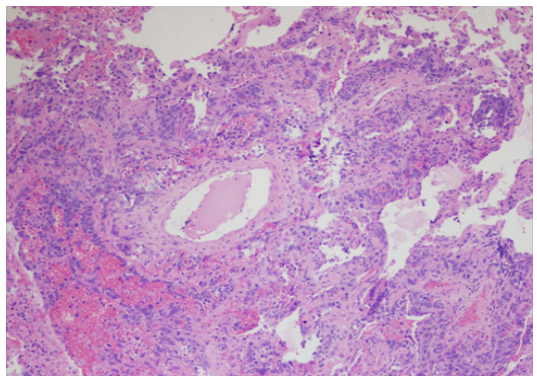

Figure 2A: Right lower lobe wedge resection. H\&E, 200x

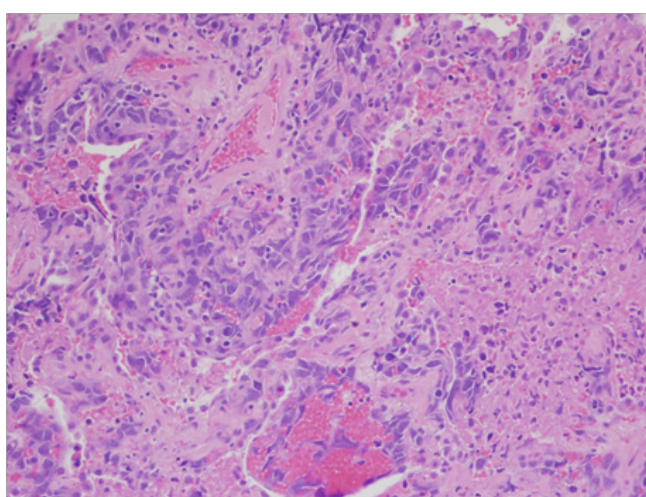

Figure 2B: Right lower lobe wedge resection. H\&E, 400x

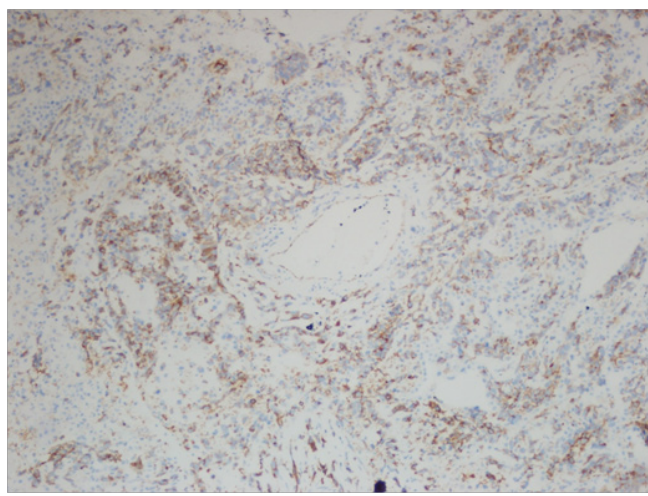

Figure 3A: Right lower lobe wedge resection. Immunohistochemical stain for CD3I, 400x.

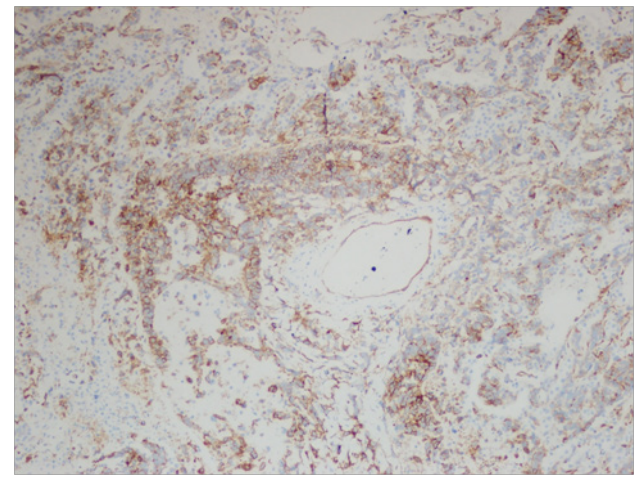

Figure 3B: Right lower lobe wedge resection. Immunohistochemical stain for CD34, 400x.

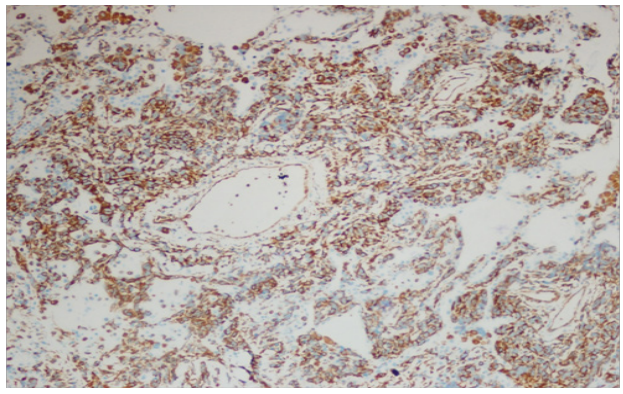

Figure 3C: Right lower lobe wedge resection. Immunohistochemical stain for vimentin, 400x. 


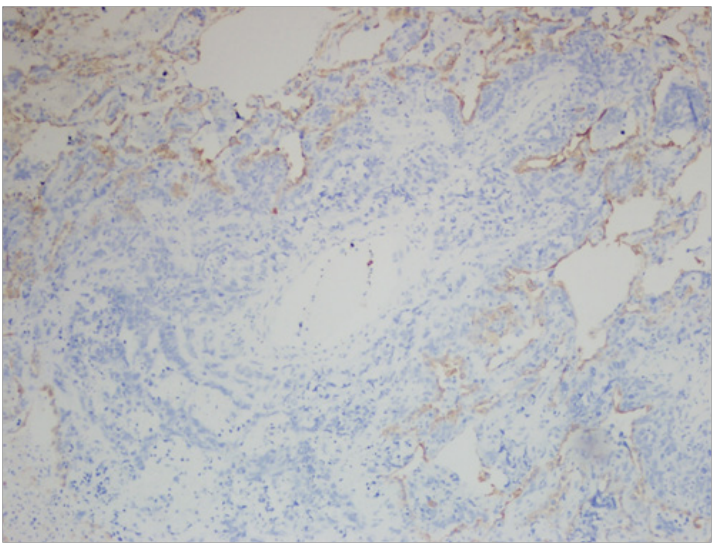

Figure 3D: Right lower lobe wedge resection. Immunohistochemical stain for AEI/AE3, 400x.

\section{Discussion}

Epidemiologic factors for cardiac angiosarcomas have not been well-characterized, however, there have been well-documented associations for other angiosarcomas, such as exposure to vinyl chloride, arsenic, thorotrast, radiotherapy and chronic lymphedema. ${ }^{2}$ More recently, angiosarcomas have been associated with a history of a chronic expanding hematoma. ${ }^{3}$

This case is dramatic in its advanced presentation, with the patient reporting a relatively short interval of symptoms of only one month. Cases of cardiac angiosarcomas can present late due to nonspecific presenting symptoms and a low index of suspicion. Most symptoms of cardiac angiosarcomas can be attributed to overwhelmingly more common cardiopulmonary conditions, including pulmonary embolism, heart failure, myocardial infarction, and autoimmunerelated effusions.

For our case, initial clinical differentials included infectious versus autoimmune, due to the patient's history o of working overseas. Tuberculosis and autoimmune causes were considered, however associated testing was negative. When considering neoplastic possibilities, the initial differential from the clinical team included myxoma and rhabdomyosarcoma. There has been a previous case of cardiac angiosarcoma misdiagnosed as rhabdomyosarcoma. ${ }^{4}$

Because of the variable symptoms and rarity of the tumor, diagnosis could be delayed. There are no specific blood biomarkers. Beneficial non-invasive tests include echocardiography, CT scanning and MRI. In patients with associated pericardial fluid, cytologic examination of pericardiocentesis may be attempted but rarely yields a definitive diagnosis. ${ }^{5}$ Tissue specimens may be obtained by transthoracic echocardiography or CT-guided biopsy, or endomyocardial biopsy, or by limited thoracotomy. ${ }^{6}$ However endomyocardial biopsy of the right atrium should be avoided as it is a thin walled structure. ${ }^{6}$

The most common anatomic location of a cardiac angiosarcoma is in the right heart, involving the atrioventricular groove, and it often encases the right coronary artery. Rarer locations include the left atrium. ${ }^{7}$ The most common metastasis is to the lungs, corresponding to hematogenous spread of tumor from the right side of the heart. Other metastatic sites described in the literature include liver, ${ }^{8}$ bones,,${ }^{9}$ lymph nodes, ${ }^{10}$ pleura, peritoneum and gastrointestinal tract. ${ }^{11}$
The histology of this tumor has been described as irregular, anastomosing, vascular structures composed of endothelial-like cells, which can be either spindle-shaped or rounded and polygonal. Low grade lesions are composed of single- or multiple-layered vascular spaces while high grade lesions show sheets of poorly differentiated, pleomorphic cells. ${ }^{2}$ Immunohistochemical stain for CD31 is positive in $90 \%$ of PCAs and stain for FLI-1 in $100 \%$ of PCAs. Other commonly used positive stains include CD34, cytokeratins, vimentin and Factor VIII-related antigen, ${ }^{12}$ ranging from $35 \%$ to $74 \%$ positivity in PCAs described in the literature.

Ancillary studies for cardiac angiosarcomas have not been wellcharacterized. Angiosarcomas in general are heterogeneous in their genetic alterations. ${ }^{13}$ For primary cardiac angiosarcoma, recent molecular studies have shown a role for mutations in the PLCG1 gene. ${ }^{14}$ The POT1 gene has also been implicated in PCA occurring in TP53-negative Li-Fraumeni-like families..$^{15}$ In breast angiosarcoma, $50 \%$ of tumor have been found to be positive for vascular endothelial growth factor receptor, ${ }^{16}$ but testing of one PCA case for EGFR was negative. ${ }^{17}$

Evidence-based treatment recommendations have not been established due to the rarity of this tumor. Both surgery and chemotherapy have been described, however none with definitive, much less curative results. Tumors involving the right heart have been treated with partial cardiectomy and Fontan cavopulmonary shunt ${ }^{18}$ and, in cases involving the left heart, auto-transplantation with reconstruction of the left atrium with bovine pericardium. ${ }^{19}$ Heart transplantation has also been described, although with limited results. ${ }^{20}$

Although surgical resection is first-line therapy, the majority of cases are metastatic upon presentation and require a multimodality approach with radiation and chemotherapy. A common regimen found in the literature is gemcitabine-docetaxel. ${ }^{11,17,21}$ Vascular endothelial growth factor receptor has been described to be present in angiosarcomas of the breast and more often in well-differentiated cases. ${ }^{16}$ One case of cardiac angiosarcoma showed focal positivity for C-KIT and the patient received imatinib for a short time, ${ }^{17}$ although its effect is unclear, as the patient also received other chemotherapeutic agents. The overall prognosis of primary cardiac angiosarcoma is poor, with survival measured in months. Death usually results from heart failure and the effects of distant metastases.

\section{Acknowledgements}

None.

\section{Conflict of interest}

The author declares no conflict of interest.

\section{References}

1. Look Hong NJ, Pandalai PK, Hornick JL, et al. Cardiac Angiosarcoma management and outcomes: 20-year single-institution experience. Ann Surg Oncol. 2012;19(8):2707-2715.

2. Andersen NJ, Froman RE, Kitchell BE. et al. Clinical and Molecular Biology of Angiosarcoma Soft Tissue Tumors, Prof. Fethi Derbel editor. In Tech; 2011.

3. Burgert-Lon CE, Riddle ND, Lackman RD, et al. Angiosarcoma arising in chronic expanding hematoma: five cases of an underrecognized association. Am J Surg Pathol. 2015;39(11):1540-1547. 
4. Gajewski P, Błaszak-Ciećwierska W, Chojnowski S, et al. Angiosarcoma of the heart - a diagnostic pitfall. Kardiol Pol. 2013;71(12):1296-1298.

5. Randall MB, Geisinger KR. Angiosarcoma of the heart: pericardial fluid cytology. Diagn Cytopathol. 1990;6(1):58-62.

6. Brandt RR, Arnold R, Bohle RM, et al. Cardiac angiosarcoma: case report and review of the literature. $Z$ Kardiol. 2005;94(12):824-828.

7. Baran C, Durdu S, Eryilmaz S, et al. Primary left cardiac Angiosarcoma with mitral valve involvement accompanying coronary artery disease. Case Rep Surg. 2015;2015:810306.

8. Qiu ZX, Zhang Q. Primary cardiac Angiosarcoma confirmed by multimodality imaging guided liver biopsy. Int $J$ Clin Exp Pathol. 2014;7(3):1188-1192.

9. Tokmak H, Demir N, Demirkool MO. Cardiac Angiosarcoma: utility of [18F] fluorodeoxyglucose positron emission tomography-computed tomography in evaluation of residue, metastases, and treatment response. Vasc Health and Risk Manag. 2014;10:399-401.

10. Jalalian R, Naghshvar F, Habibi V, et al. Primary cardiac angiosarcoma in a middle aged woman. IranRed Crescent Med J. 2015;17(6):e23067.

11. Wang $\mathrm{M}, \mathrm{Fu} \mathrm{G}$, Jiang $\mathrm{H}$, et al. Multimodality treatment for cardiac Angiosarcoma. Intern Med. 2014;53(17):1949-1953.

12. Patel SD, Peterson A, Bartczak A, et al. Primary cardiac Angiosarcoma - a review. Med Sci Monit. 2014;20:103-109.
13. Murali R, Chandramohan R, Moller I, et al. Targeted massively parallel sequencing of angiosarcomas reveals frequent activation of the mitogen activated protein kinase pathway. Oncotarget. 2015;6(34):36041-36052.

14. Kunze K, Spieker T, Gamerdinger U, et al. A recurrent activating PLCG1 mutation in cardiac Angiosarcomas increases apoptosis resistance and invasiveness of endothelial cells. Cancer Res. 2014;74(21):6173-6183.

15. Calvete O, Martinez P, Garcia-Pavia P, et al. A mutation in the POT1 gene is responsible for cardiac Angiosarcoma in TP53-negative Li-Fraumenilike families. Nat Commun. 2015;6:8383.

16. Gennaro M, Valeri B, Casalini P, et al. Angiosarcoma of the breast and vascular endothelial growth factor receptor. Tumori. 2010;96(6):930-935.

17. Bellitti R, Buonocore M, De Rosa N, et al. Primary cardac Angiosarcoma in a 25-year-old man. Tex Heart Inst J. 2013;40(2):186-188.

18. Hoffmeier A, Deiters S, Schmidt G, et al. Radical resection of cardiac sarcoma. Thorac Cardiovasc Surg. 2004;52(2):77-81.

19. Conklin LD, Reardon MJ. Cardiac Autotransplantation for Primary Cardiac Malignancy. Tex Heart Inst J. 2002;29(2):105-108.

20. Uberfuhr PM, Meiser B, Fuchs A, et al. Heart transplantation: An approach to treating primary cardiac sarcoma? J Heart Lung Transplant. 2002;21(10):1135-1139.

21. Chalhoub E, Mattar BI, Shaheen W, et al. Cardiac Angiosarcoma presenting with tamponade. Intern Med. 2012;5(20):2905-2907. 